\title{
Inertização de zinco presente em resíduo perigoso por meio do uso de produtos industriais
}

\section{Inertization of zinc contained in hazardous waste by application of industrial products}

\author{
1 Ana Carolina Callegario Pereira \\ 2 André Marques dos Santos \\ 3 Danielle França de Oliveira \\ 1 Camila da Costa Barros \\ 1 Nelson Mazur \\ 1 Centro Universitário de Volta Redonda - UniFOA
2 Universidade Federal Rural do Rio de Janeiro - UFRRJ
3 Universidade Federal Fluminense - UFF
}

\section{RESUMO}

Com o objetivo de reduzir o potencial poluente de um resíduo ácido contaminado proveniente da Cia Mercantil e Industrial Ingá, depositado de forma inadequada às margens da Baía de Sepetiba - Itaguaí - RJ, foram testados a capacidade de ação de três produtos industriais na inertização de metais pesados. 0 experimento foi conduzido em delineamento experimental inteiramente casualizado, com três repetições, totalizando trinta unidades experimentais. Os tratamentos utilizados foram: Resíduo Contaminado (Resíduo Ingá) (Testemunha), Resíduo Contaminado + Produto 1 (0,5\%), Resíduo Contaminado + Produto 1 (2,0\%), Resíduo Contaminado + Produto 1 (5,0\%), Resíduo Contaminado + Produto 2 (0,9\%), Resíduo Contaminado + Produto 2 (2,4\%), Resíduo Contaminado + Produto 2 (3,8\%), Resíduo Contaminado + Produto 3 (0,3\%), Resíduo Contaminado + Produto 3 (1,2 $\%)$ e Resíduo Contaminado + Produto 3 (2,2 \%). Durante o experimento, foram retiradas amostras de cada tratamento nos tempos de 0, 20, 40 e 60 dias e, em cada amostra, foram realizadas determinações de $\mathrm{pH}(1: 2,5)$ e extrações simples. A partir das concentrações de Zn obtidas nas extrações, estimou-se as concentrações de Zn nas diferentes formas químicas. Os inertizantes utilizados foram capazes de reduzir a solubilidade do Zn, reduzindo seus efeitos maléficos ao ambiente.

\section{Palavras-chave}

Resíduo. Contenção química. Extração simples.

\begin{abstract}
For the purpose of reducing the pollution risk of an acid-contaminated residue from the productive activities of Cia. Mercantil e Industrial Ingá, deposited in inadequate form along the banks of Sepetiba Bay, Itaguaí, Rio de Janeiro, we tested the ability of three industrial products to render heavy metals inert. The experimental design was fully randomized, with three repetitions and a total of 30 experimental units. The treatments used were: Contaminated Waste (Ingá Waste - control), Contaminated Waste + Product 1 (0.5\%), Contaminated Waste + Product 1 (2.0\%), Contaminated Waste + Product 1 (5.0\%), Contaminated Waste + Product 2 (0.9\%), Contaminated Waste + Product 2 (2.4\%), Contaminated Waste + Product 2 (3.8\%), Contaminated Waste + Product 3 (0.3\%), Contaminated Waste + Product 3 (1.2\%) and Contaminated Waste + Product 3 (2.2\%). During the experiment, samples were retrieved from each treatment on days 0,20, 40 and 60 . Each sample was analyzed to measure the $\mathrm{pH}(1: 2.5)$ and for simple extractions. From the concentrations of $\mathrm{Zn}$ in the extractions, we estimated the concentrations of $\mathrm{Zn}$ in different chemical forms. The intertizers used were able to reduce the solubility of $\mathrm{Zn}$, diminishing its harmful effects on the environment.
\end{abstract}

\section{Keywords}

Waste. Chemical containment. Simple extractions.

\section{Como você deve citar?}

PEREIRA, Ana Carolina Callegario et al. Inertização de zinco presente em resíduo perigoso por meio do uso de produtos industriais. Cadernos UniFOA, Volta Redonda, n. 35, p. 25-34, dez. 2017. 


\section{INTRODUÇÃO}

O crescimento populacional das sociedades de consumo resulta em um considerável aumento na produção de resíduos e consequente contaminação de áreas próximas a complexos industriais, urbanos e áreas de agricultura altamente tecnificadas, devido ao depósito de substâncias resultantes dessas atividades (RODRIGUES, 2010). A aplicação de resíduos urbanos e industriais e do uso de fertilizantes e pesticidas na agricultura são as principais atividades que originam a contaminação do solo (MELO et al. 2006; TELES e FERREIRA, 2017).

A classificação de resíduos sólidos é realizada de acordo com a NBR 10004: 2004, que classifica os resíduos sólidos quanto aos seus riscos potenciais ao meio ambiente e à saúde pública, visando seu gerenciamento adequado (ABNT, 2004). Essa classificação é realizada conforme suas características específicas, tais como toxicidade, inflamabilidade, corrosividade, reatividade e sua atividade de origem (AUGUSTO et al., 2012)

Em função das características específicas dos resíduos, esses resíduos exigem cuidados especiais quanto ao manuseio, acondicionamento, coleta, transporte e destino final, a fim de se evitar que causem danos ao ambiente. Dentre esses contaminantes, destacam-se os metais pesados que, por serem bastante estáveis na natureza, são passíveis de serem acumulados no solo e em sistemas biológicos ao longo do tempo (RODRIGUES, 2010).

Os metais pesados incorporados no solo podem seguir diferentes vias de fixação, liberação ou transporte, podendo ficar retidos no solo, sejam dissolvidos em solução ou fixados por processos de adsorção, complexação e precipitação (MARQUES, 2011).

A Cia. Mercantil e Industrial INGÁ, localizada no município de Itaguaí-RJ, é considerada a maior poluidora da região. Foi uma usina produtora de metal primário Zinco (Zn), onde ocorreram atividades de mineração, redução e refino do metal, por aproximadamente 33 anos.

A extração de $Z n$ a partir do minério de calamina era feita pelo processo via úmida ou hidrometalurgia, no qual o princípio fundamental era produzir um composto solúvel e lixiviável, e separar da lixívia as impurezas, obtendo-se uma solução purificada para fazer a eletrólise. Os produtos obtidos eram o zinco puro e o ácido sulfúrico, sendo o último reutilizado como solvente. No processo de extração do metal, era produzido um resíduo sólido rico em impurezas contidas no minério e também obtidas por meio do processo de extração do zinco, como os metais pesados Cadmio (Cd), Chumbo (Pb), Manganês (Mn), Níquel (Ni), Cobalto (Co), Arsênio (As), Zn, entre outros. 0 resíduo era disposto no pátio da indústria, sem condições apropriadas de armazenamento, aumentando os riscos de contaminação do meio ambiente, principalmente com Zn.

A melhoria das condições químicas do Resíduo Ingá, de forma a reduzir a solubilidade dos metais, pode ser uma alternativa importante para a remediação das áreas impactadas na região próxima à Cia. Ingá. Dessa forma, este trabalho teve por objetivo verificar a eficiência do uso de produtos industriais na inertização do metal Zn presente no Resíduo Ingá. 


\section{MATERIAL E MÉTODOS}

\subsection{Caracterização do Resíduo Ingá}

A amostra de resíduo utilizada foi coletada em abril de 2002. A coleta consistiu em retirar amostras simples do topo, meio e base da pilha de resíduo mantida no pátio de armazenamento da indústria, formando uma amostra composta de $100 \mathrm{~kg}$, segundo a norma 10007 (ABNT, 2004). No laboratório, a amostra do resíduo foi seca ao ar, destorroada e passada por peneira de $2 \mathrm{~mm}$ e acondicionada em sacos plásticos.

\subsection{Resíduo Ingá}

Na tabela 1, observa-se que os teores totais de Zn, Cd, Mn, Pb e Fe são elevados. Santos et al. (2005) demonstram que, a partir de testes de solubilidade e lixiviação realizados de acordo com as normas da ABNT (2004), o resíduo é classificado como Perigoso.

Tabela 1 - Teores totais, solúveis e lixiviáveis de $\mathrm{Zn}, \mathrm{Cd}, \mathrm{Mn}, \mathrm{Pb}, \mathrm{Fe}, \mathrm{Cu}, \mathrm{Ni}$ no resíduo da Ingá $\left(\mathrm{mg} \mathrm{kg}^{-1}\right)$

\begin{tabular}{|c|c|c|c|c|c|c|c|}
\hline Fração & Zn & Cd & Mn & $\mathrm{Pb}$ & $\mathrm{Fe}$ & $\mathrm{Cu}$ & $\mathrm{Ni}$ \\
\hline Total & 22294,4 & 175,2 & 5525,6 & 2486,8 & 79986,7 & 123,2 & 32,0 \\
\hline Teste de solubilidade & $\begin{array}{l}1755 \\
(7,9)\end{array}$ & $\begin{array}{l}31,7 \\
(18)\end{array}$ & $\begin{array}{l}34,1 \\
(0,6)\end{array}$ & $\begin{array}{l}0,93 \\
(0,04)\end{array}$ & $\begin{array}{l}0,13 \\
(0)\end{array}$ & $\begin{array}{l}0,10 \\
(0,08)\end{array}$ & $\begin{array}{l}1,04 \\
(3,2)\end{array}$ \\
\hline Teste de lixiviação & & 43,4 & & 1,60 & & & \\
\hline
\end{tabular}

Os números entre parênteses correspondem à percentagem em relação ao total.

Fonte: Santos et al. (2005)

\subsection{Caracterização dos produtos industriais (Inertizantes)}

A coleta desses produtos consistiu em retirar amostras do topo, meio e base da pilha de resíduos, formando uma amostra composta de $30 \mathrm{~kg}$ (ABNT, 2004), exceto o Produto 3, que foi obtido em embalagem comercial. Em laboratório, foi realizado o mesmo procedimento aplicado ao Resíduo Ingá.

Produto 1: É um resíduo inerente ao processo de fabricação de borracha sintética (SBR), uma vez que o látex, produto da reação de copolimerização do butadieno e do estireno, fica aderido às paredes dos vasos de processo. Sua destinação atual é a incineração, o que gera elevado custo para a indústria (Tabela 2).

Produto 2: É um resíduo alcalino que consiste em uma biomassa obtida pela coagulação e precipitação do material proteico celular dos lactobacilos utilizados na fabricação de ácido láctico, ao se alcalinizar o mosto fermentado, com um excesso de cal à temperatura de $80^{\circ} \mathrm{C}$. 0 material é separado por filtração em filtro rotativo a vácuo, utilizando-se gesso como pré-camada e que a ele se incorpora (Tabela 2).

Produto 3: Carbonato de Cálcio $\left(\mathrm{CaCO}_{3}\right)$ - Reagente PA. Produto alcalino. 
Tabela 2 - Algumas características dos resíduos utilizados.

\begin{tabular}{lll}
\hline Características & Produto 1 & Produto 2 \\
\hline Forma & Sólida & Sólida \\
Cor & Cinza Escuro & Creme \\
Umidade & $16,7 \%$ & $70-80 \%$ \\
pH H2O & 5,1 & $\mathbf{1 0 , 5}-\mathbf{1 2 , 5}$ \\
Matéria Orgânica Total & $\mathbf{8 , 4} \%$ & -- \\
$\mathrm{Cd}$ & $<0,001 \mathrm{mg} \mathrm{k}^{-1}$ & $0 \mathrm{mg} \mathrm{kg} 1$ \\
$\mathrm{Zn}$ & $<0,1 \mathrm{mg} \mathrm{k}^{-1}$ & $10 \mathrm{mg} \mathrm{kg} 1$ \\
\hline Análise realizada: & Hidroquímica Engenharia e & Centro de Análises da UFRRJ, Campus \\
& Laboratórios LTDA. & DR. Leonel Miranda \\
\hline
\end{tabular}

\section{CONDUÇÃO DO EXPERIMENTO}

O experimento foi instalado em recipientes plásticos, contendo $150 \mathrm{~g}$ da mistura dos resíduos, umedecidos a $80 \%$ da capacidade de campo, permanecendo incubados por um período de 60 dias, para posteriormente se avaliar a capacidade dos produtos industriais inertizarem o Zn contido no Resíduo Ingá.

Os tratamentos utilizados foram: Resíduo contaminado (Resíduo Ingá) (testemunha), Resíduo contaminado + Produto 1 (0,5\%), Resíduo contaminado + Produto 1 (2,0\%), Resíduo contaminado + Produto 1 (5,0\%), Resíduo contaminado + Produto $2(0,9 \%)$, Resíduo contaminado + Produto $2(2,4 \%)$, Resíduo contaminado + Produto 2 (3,8\%), Resíduo contaminado + Produto 3(0,3\%), Resíduo contaminado + Produto 3 (1,2\%) e Resíduo contaminado + Produto 3(2,2 \%).

Foram retiradas amostras de cada tratamento em intervalos de 0,20, 40, 60 dias, para a determinação do pH em água $(1: 2,5)$ e realização das extrações simples. As extrações simples foram realizadas, utilizando-se os seguintes extratores: Água (MILLER et al., 1986; DO NASCIMENTO et al., 2014), Nitrato de sódio (KELLER \& VÉDY, 1994) (ZHU et al., 2012), DTPA (LINDSAY \& NORWELL, 1978) (DO NASCIMENTO et al., 2014) e Ácido acético (URE et al., 1993; ARAUJO et al., 2011).

Para todas as extrações, foram determinados os brancos, que consistiram em extrações sem os tratamentos. Todas as extrações foram realizadas com 3 repetições.

\subsection{Determinação das formas químicas de metais pesados}

A partir dos resultados obtidos por meio das extrações simples, foram estimadas as frações químicas de Zn, através das diferenças nas quantidades extraíveis:

$\mathrm{F}_{1}=$ Extraído com Água (Fração solúvel em água); $\mathrm{F}_{2}=$ Extraído com $\mathrm{NaNO}_{3}$ - Extraído com Água (Fração trocável), que compreende os metais ligados eletrostaticamente às partículas do solo; $\mathbf{F}_{3}=$ Extraído com DTPA - Extraído com $\mathrm{NaNO}_{3}$ (Fração predominantemente associada a óxidos de Fe e Mn mais cristalinos e a compostos orgânicos mais estáveis através da formação de complexos de esfera interna); $\mathrm{F}_{4}=$ Extraído com ácido acético - Extraído com $\mathrm{NaNO}_{3}$ (Fração associada predominantemente a carbonatos e a óxidos de manganês amorfos pela formação de complexos de transição); $F_{5}=$ Total $-\Sigma(F 1+F 2+F 3+F 4)$ (Fração associada a compostos de alto grau de cristalinidade, fração residual).

Os dados obtidos foram analisados estatisticamente, utilizando-se o programa SAEG versão 5.0, e as médias foram comparadas pelo teste de Tukey a $5 \%$ de significância de acordo com a diferença mínima significativa. 


\section{RESULTADOS E DISCUSSÃO}

\subsection{Efeito das diferentes doses de cada inertizante no pH do Resíduo Ingá}

$\mathrm{O} \mathrm{pH}$ do solo é um fator que influencia diretamente na disponibilidade dos metais no solo por meio do aumento da CTC do solo. Em geral, ambientes ácidos determinam uma maior mobilidade do metal, enquanto condições de $\mathrm{pH}$ acima de seis favorecem a processo de retenção, principalmente em solos com elevado grau de intemperização, onde os grupos funcionais de superfície dos componentes coloidais são, na sua maioria, pH dependentes (oxihidróxidos de ferro e alumínio) (DE OLIVEIRA et al., 2011). Desse modo, a manipulação o pH é um método eficaz para o controle da biodisponibilidade e a mobilidade de metais pesados em solos.

Ao final do experimento, foi observado que os valores de $\mathrm{pH}$ na testemunha permaneceram constantes em todos os tempos de incubação (Tabela 3). Apesar desses valores não terem sofrido alteração em condições controladas, não se pode afirmar que o mesmo acontece no montante de resíduo acumulado no pátio da Cia. Ingá, a céu aberto, devido à ocorrência de precipitação (chuvas), que poderia causar variação no potencial hidrogeniônico $(\mathrm{pH})$.

Foi observado que a dose $(0,5 \%)$ do Produto 1 não sofreu efeito do tempo, provavelmente por oferecer baixa contribuição de matéria orgânica ao sistema, não tendo sido relevante a ponto de causar variações no $\mathrm{pH}$. As doses $(2,0 \%)$ e $(5,0 \%)$ do Produto 1 foram capazes de proporcionar maiores valores de $\mathrm{pH}$ nos maiores tempos de incubação. 0 mesmo aconteceu para o Produto 3 , no entanto, para o Produto 2, a única dose capaz de causar variação foi a dose (0,9\%) (Tabela 3$)$.

A dose $(0,9 \%)$ do Produto 2 foi a única a apresentar variações ao longo dos tempos de incubação, possivelmente por ser a menor dose em relação às demais, possibilitando ainda a ocorrência de reações até que se alcance a estabilidade nos valores de $\mathrm{pH}$. As doses $(2,4 \%)$ e $(3,8 \%)$ do Produto 2 foram capazes de alcançar estabilidade nos valores de $\mathrm{pH}$ no tempo 1.

O Produto 3, assim como o Produto 2, é alcalino, entretanto observa-se comportamento diferenciado em relação às variações ocorridas ao longo dos tempos de incubação, o que pode ser explicado pela diferença na composição desses produtos. A dose $(0,3 \%)$ do Produto 3 não apresentou variação significativa ao longo dos tempos de incubação. Esse comportamento pode ser explicado pela diferença na velocidade de reação Inertizante - Inertizado, que deve ter sido maior no caso da utilização do Produto 3, fazendo com que se atingisse uma estabilidade nos valores de $\mathrm{pH}$, já na menor dose.

Portanto, os maiores valores de $\mathrm{pH}$ foram encontrados nas maiores doses dos Produtos 2 e 3 . 
Tabela 3 - Valores médios de pH, ao longo dos tempos de incubação nos diferentes tratamentos.

\begin{tabular}{|c|c|c|c|c|c|c|c|c|c|c|}
\hline \multirow{3}{*}{$\begin{array}{l}\text { Tempo } \\
\text { (dias) }\end{array}$} & TESTE & \multicolumn{3}{|c|}{ Produto 1} & \multicolumn{3}{|c|}{ Produto 2} & \multicolumn{3}{|c|}{ Produto 3} \\
\hline & \multicolumn{10}{|l|}{ Doses\% } \\
\hline & 0,0 & 0,5 & 2,0 & 5,0 & 0,9 & 2,4 & 3,8 & 0,3 & 1,2 & 2,2 \\
\hline 0 & $5,9 \mathrm{aEF}$ & $5,9 \mathrm{aEF}$ & $5,7 \mathrm{bF}$ & 6,0abDE & $5,8 \mathrm{bF}$ & $6,1 \mathrm{aCD}$ & $6,5 \mathrm{aB}$ & $6,0 \mathrm{aDE}$ & $6,2 \mathrm{bC}$ & $6,8 \mathrm{cA}$ \\
\hline 20 & $5,9 \mathrm{aDE}$ & $5,8 \mathrm{aE}$ & $6,1 \mathrm{aCDE}$ & $6,0 \mathrm{bCDE}$ & $5,9 \mathrm{aDE}$ & $6,1 \mathrm{aCDE}$ & $6,5 \mathrm{aB}$ & $6,3 \mathrm{aBC}$ & $6,2 \mathrm{bCD}$ & $7,1 \mathrm{bcA}$ \\
\hline 40 & $6,0 \mathrm{aB}$ & $5,9 \mathrm{aB}$ & $6,0 \mathrm{aB}$ & $5,9 \mathrm{cB}$ & $5,9 a \mathrm{~B}$ & $6,1 \mathrm{aB}$ & $6,5 \mathrm{aAB}$ & $5,9 a B$ & $6,3 a B$ & $7,2 \mathrm{bA}$ \\
\hline 60 & $5,9 \mathrm{aCD}$ & $5,7 \mathrm{aD}$ & $6,0 \mathrm{aCD}$ & $6,0 \mathrm{aCD}$ & 5,8abCD & $6,1 \mathrm{aCD}$ & $6,6 \mathrm{aB}$ & $6,0 \mathrm{aCD}$ & $6,2 \mathrm{abBC}$ & $7,4 \mathrm{aA}$ \\
\hline
\end{tabular}

Valores seguidos pela mesma letra não diferem significativamente pelo teste de Tukey ao nível de $5 \%$ de probabilidade. As letras maiúsculas comparam as doses horizontalmente. As letras minúsculas comparam os tempos de incubação verticalmente.

\subsection{Distribuição do elemento Zn nas diferentes frações químicas.}

Com o objetivo de reduzir a solubilidade do elemento Zn presente no Resíduo Ingá, foram aplicados três produtos industriais na inertização de metais pesados: Produto 1 (com característica orgânica), Produto 2 e Produto 3 (com característica alcalina).

É conhecido que as reações de precipitação, complexação e adsorção de metais pesados são favorecidas pela elevação de pH (SPOSITO, 2008; LEE et al., 2017), assim como a diminuição da acidez do sistema solo-resíduo alcalino pode favorecer a transformação de Zn nas formas mais solúveis para as de menor solubilidade. Os metais pesados apresentam maior solubilidade em condições de acidez, e aumentos do $\mathrm{pH}$ do solo reduzem a sua disponibilidade para as plantas (BORGES \& COUTINHO, 2004; LEE et al., 2017). Estudos demonstraram que a adsorção específica do $\mathrm{Zn}$ pelos minerais do solo aumenta com o pH (ANDRADE et al., 2007; SANTOS, 2005; BORGES JUNIOR et al., 2008; UDDIN, 2017; KWON et al., 2017), o que indicaria uma eficiência na redução da solubilidade do Zn presente no Resíduo Ingá.

Além da alcalinidade, a ação da matéria orgânica é importante na inertização de metais pesados, já que a matéria orgânica forma complexos solúveis (quelatos) com os metais pesados, e isso acontece através das moléculas orgânicas solúveis de baixo peso molecular que são produzidas durante a decomposição microbiana do resíduo orgânico no solo. Na decomposição da matéria orgânica, o produto secundário formado é o húmus, um material com propriedades coloidais e com alta capacidade de troca de ânions. O húmus é a parte mais estável da matéria orgânica que atinge o estado coloidal com alta densidade de cargas elétricas em sua superfície, sendo capaz de adsorver e trocar cátions, o que faz com que pequenas quantidades aumentem muito as características dinâmicas do contaminante (LEPSCH, 2010).

A matéria orgânica possui afinidade pelos metais pesados presentes no solo, tal afınidade apresenta-se de duas formas: a matéria orgânica proporciona sítios de adsorção (específicos ou não), sendo, portanto similar à adsorção de argilas e hidróxidos, e atua também como agente quelante do metal na solução do solo, tais formas ocorrem em razão da sua configuração e profusão de grupos fenólicos e carboxílicos por ela apresentada (SIMÃO \& SIQUEIRA, 2001).

Sendo assim, compostos orgânicos simples são efetivos complexantes ou agentes quelantes para o $\mathrm{Zn}$, o que aumenta a mobilidade e solubilidade desse metal no solo. Esse mecanismo ocorre mais frequentemente com Fe, Zn, Cu e Mn (SANTOS, 2005; ZHOU et al., 2018). 
As maiores doses dos inertizantes 1, 2 e 3 proporcionaram menores valores de $Z n$ na F1, e maiores valores de Zn encontrados na fração mais estável (F5) (Tabela 4).

Esse resultado pode ser explicado através dos constituintes de cada produto utilizado, pois a eficiência da matéria orgânica está relacionada à presença de substâncias húmicas, constituídas de ácidos fúlvicos, ácidos húmicos e humina, que, sob a denominação de húmus, representam os principais agentes orgânicos envolvidos na complexação de metais no solo (SIMÃO \& SIQUEIRA, 2001).

A ação da alcalinidade é também comprovadamente eficiente (XU et al., 2017). Em solos com reação variando de neutra a alcalina, os metais pesados tornam-se menos solúveis e menos disponíveis às plantas, por formarem precipitados com hidróxidos e carbonatos (NASCIMENTO et al., 2002). Outra consequência do $\mathrm{pH}$ na disponibilidade dos metais no solo é o aumento da CTC do solo, o que favorece a adsorção de cátions, sendo esse efeito mais pronunciado em solos com carga variável (SIMÃO \& SIQUEIRA, 2001). Nascimento et al. (2002) observaram que, na presença de resíduos alcalinos, ocorre uma drástica redução na fração Zn-trocável. Dessa forma, esta encontra-se inversamente relacionada ao $\mathrm{pH}$.

Todos os inertizantes utilizados foram eficientes na redução da biodisponibilidade do $\mathrm{Zn}$, visto que os maiores teores do elemento passaram de frações menos estáveis para frações mais estáveis no decorrer do experimento, ocorrendo uma redução da fração $\mathrm{F} 1 \mathrm{e}$, consequentemente, um aumento na fração F4.

Tabela 4 - Valores percentuais de Zn nas diferentes frações químicas e tratamentos.

\begin{tabular}{|c|c|c|c|c|c|c|c|c|c|c|}
\hline \multirow{2}{*}{$\begin{array}{l}\text { Tempos de } \\
\text { Incubação }\end{array}$} & \multirow[t]{2}{*}{ Testemunha } & \multicolumn{3}{|l|}{ Produto 1} & \multicolumn{3}{|l|}{ Produto 2} & \multicolumn{3}{|l|}{ Produto 3} \\
\hline & & $0,5 \%$ & $2,0 \%$ & $5,0 \%$ & $0,9 \%$ & $2,4 \%$ & $3,8 \%$ & $0,3 \%$ & $1,2 \%$ & $2,2 \%$ \\
\hline & Fração F1 & & & & & & & & & \\
\hline 0 Dia & $20,9 \mathrm{bA}$ & $21,0 \mathrm{bA}$ & $17,8 \mathrm{aAB}$ & 2,2aCB & $20,8 \mathrm{abA}$ & $14,4 \mathrm{cB}$ & $14,5 a b B$ & $20,0 \mathrm{bAB}$ & $7,1 \mathrm{aC}$ & $0,1 \mathrm{aD}$ \\
\hline 20 Dias & $30,0 \mathrm{aA}$ & $31,2 \mathrm{aA}$ & $15,8 \mathrm{aC}$ & $0,2 \mathrm{aD}$ & $25,9 a A B$ & $27,5 a A B$ & $16,6 \mathrm{aC}$ & $19,8 b B C$ & $2,9 a D$ & $0,1 \mathrm{aD}$ \\
\hline 40 Dias & $24,3 a b A B$ & $27,8 \mathrm{abA}$ & $18,5 \mathrm{aABC}$ & $0,2 \mathrm{aD}$ & $19,0 \mathrm{bABC}$ & $15,6 \mathrm{cBC}$ & $11,0 \mathrm{bC}$ & $25,4 a A B$ & $12,0 \mathrm{aC}$ & $0,1 \mathrm{aD}$ \\
\hline \multirow[t]{2}{*}{60 Dias } & $28,0 \mathrm{abA}$ & $28,1 \mathrm{abA}$ & $15,9 \mathrm{aCD}$ & $0,2 \mathrm{aE}$ & $20,0 \mathrm{abBC}$ & $24,1 \mathrm{bAB}$ & 13,3abD & $15,1 \mathrm{bCD}$ & $0,1 \mathrm{aE}$ & $1,5 \mathrm{aE}$ \\
\hline & Fração F2 & & & & & & & & & \\
\hline 0 Dia & $2,5 \mathrm{aABC}$ & $5,6 \mathrm{cAB}$ & $4,5 \mathrm{aABC}$ & $5,8 \mathrm{abA}$ & $1,0 \mathrm{aC}$ & $1,5 \mathrm{aC}$ & $1,1 \mathrm{aC}$ & $1,8 \mathrm{aBC}$ & $1,1 \mathrm{aC}$ & $1,4 \mathrm{aC}$ \\
\hline 20 Dias & $2,8 \mathrm{aA}$ & $3,1 \mathrm{abA}$ & $4,7 \mathrm{aA}$ & $3,9 \mathrm{bA}$ & $6,0 \mathrm{aA}$ & $2,9 a A$ & $1,6 a A$ & $2,2 \mathrm{aA}$ & $5,3 a A$ & $0,4 \mathrm{aA}$ \\
\hline 40 Dias & $6,3 \mathrm{aA}$ & $1,0 \mathrm{bA}$ & $3,7 a A$ & $7,2 \mathrm{aA}$ & $7,3 \mathrm{aA}$ & $5,0 \mathrm{aA}$ & $1,9 a A$ & $2,2 \mathrm{aA}$ & $7,5 \mathrm{aA}$ & $0,3 \mathrm{aA}$ \\
\hline \multirow[t]{2}{*}{60 Dias } & $4,0 \mathrm{aAB}$ & $2,7 a b A B$ & $4,6 \mathrm{aAB}$ & $6,0 \mathrm{abA}$ & $4,7 \mathrm{aAB}$ & $3,0 \mathrm{aAB}$ & $1,2 \mathrm{aB}$ & $3,5 \mathrm{aAB}$ & $0,4 \mathrm{aB}$ & $2,0 \mathrm{aAB}$ \\
\hline & Fração F3 & & & & & & & & & \\
\hline 0 Dia & $6,7 \mathrm{aABC}$ & $5,1 \mathrm{aBCD}$ & $4,2 \mathrm{aBCD}$ & $11,2 \mathrm{bA}$ & $2,3 \mathrm{aCD}$ & $2,0 \mathrm{aCD}$ & $2,8 \mathrm{aBCD}$ & $1,2 \mathrm{aD}$ & $2,7 \mathrm{cBCD}$ & $7,4 \mathrm{bAB}$ \\
\hline 20 Dias & $2,7 \mathrm{aCD}$ & $3,1 \mathrm{aCD}$ & $6,6 \mathrm{aB}$ & $19,3 a A$ & $2,2 \mathrm{aCD}$ & $3,7 \mathrm{aCD}$ & $1,6 \mathrm{aD}$ & $1,9 \mathrm{aCD}$ & $4,9 \mathrm{bCD}$ & $10,0 \mathrm{aB}$ \\
\hline 40 Dias & $6,5 \mathrm{aBC}$ & $5,6 \mathrm{aC}$ & $5,1 \mathrm{aC}$ & $20,0 \mathrm{aA}$ & $4,5 \mathrm{aC}$ & $2,0 \mathrm{aC}$ & $1,7 \mathrm{aC}$ & $2,8 \mathrm{aC}$ & $2,0 \mathrm{cC}$ & $11,5 \mathrm{aB}$ \\
\hline \multirow[t]{2}{*}{60 Dias } & $3,3 \mathrm{aD}$ & $5,2 \mathrm{aD}$ & $2,5 \mathrm{aD}$ & $16,1 \mathrm{abA}$ & $5,8 \mathrm{aCD}$ & $2,0 \mathrm{aD}$ & $1,7 \mathrm{aD}$ & $2,5 \mathrm{aD}$ & $10,5 \mathrm{aB}$ & $10,0 \mathrm{aBC}$ \\
\hline & Fração F4 & & & & & & & & & \\
\hline 0 Dia & $33,5 \mathrm{bAB}$ & $45,6 a b A$ & $39,6 a A B$ & $31,3 \mathrm{bB}$ & $37,3 \mathrm{bAB}$ & $40,0 \mathrm{abAB}$ & $33,7 \mathrm{bAB}$ & $34,5 \mathrm{cAB}$ & $34,1 \mathrm{aAB}$ & $28,8 \mathrm{bB}$ \\
\hline 20 Dias & $43,7 \mathrm{abAB}$ & $35,6 \mathrm{bB}$ & $45,2 \mathrm{aAB}$ & $35,6 a b B$ & $41,2 \mathrm{abAB}$ & $37,3 \mathrm{bB}$ & $58,3 a A$ & $43,0 \mathrm{bcAB}$ & $43,6 a A B$ & $34,7 \mathrm{abB}$ \\
\hline 40 Dias & $40,8 b B C$ & $55,0 \mathrm{aAB}$ & $55,7 \mathrm{aAB}$ & $32,6 a b c$ & $53,0 \mathrm{abABC}$ & $56,8 \mathrm{abAB}$ & $64,4 \mathrm{aA}$ & $48,6 \mathrm{bABC}$ & $42,0 \mathrm{aBC}$ & $36,2 \mathrm{aBC}$ \\
\hline \multirow[t]{2}{*}{60 Dias } & $55,0 \mathrm{aABCD}$ & $58,1 \mathrm{aABC}$ & $51,7 \mathrm{aBCD}$ & $41,7 \mathrm{aD}$ & $62,6 a A B$ & $58,3 \mathrm{aABC}$ & $69,1 \mathrm{aA}$ & $59,2 \mathrm{aABC}$ & $44,8 \mathrm{aCD}$ & $40,1 \mathrm{aD}$ \\
\hline & Fração F5 & & & & & & & & & \\
\hline 0 Dia & $36,4 \mathrm{aCD}$ & $22,6 a b E$ & $34,0 \mathrm{aDE}$ & $49,5 \mathrm{aABC}$ & $38,7 \mathrm{aCD}$ & $42,1 \mathrm{aBCD}$ & $47,8 \mathrm{aBC}$ & $42,4 \mathrm{aBCD}$ & $55,0 \mathrm{aAB}$ & $62,4 \mathrm{aA}$ \\
\hline 20 Dias & $20,7 \mathrm{bD}$ & $26,9 \mathrm{aBCD}$ & $27,8 \mathrm{aBCD}$ & $41,0 \mathrm{bABC}$ & $24,6 \mathrm{abCD}$ & $28,6 a b B C D$ & $22,0 \mathrm{bD}$ & $33,1 \mathrm{aBCD}$ & $43,3 \mathrm{aAB}$ & $54,8 \mathrm{abA}$ \\
\hline 40 Dias & $22,2 \mathrm{bBCD}$ & 10,6bcD & $17,0 \mathrm{aD}$ & $40,1 \mathrm{bAB}$ & $16,3 b D$ & $20,6 b C D$ & $18,1 b D$ & $21,0 \mathrm{bCD}$ & $36,4 \mathrm{aABC}$ & $52,0 \mathrm{bcA}$ \\
\hline 60 Dias & $9,7 \mathrm{cDE}$ & $6,0 \mathrm{cE}$ & $25,3 a B C$ & $36,0 \mathrm{bAB}$ & $7,0 \mathrm{bE}$ & $12,6 \mathrm{bDE}$ & $14,7 \mathrm{bCDE}$ & $19,7 \mathrm{bCD}$ & $44,2 \mathrm{aA}$ & $46,4 \mathrm{cA}$ \\
\hline
\end{tabular}

Fonte: próprio autor. 


\section{CONCLUSÕES}

- $\mathrm{O} \mathrm{pH}$, devido a sua influência na biodisponibilidade do $\mathrm{Zn}$, é um fator que deve ser considerado, no que diz respeito à solubilidade do contaminante. No presente estudo, os valores de $\mathrm{pH}$ da Testemunha e da dose $(0,5 \%)$ do Produto 1 , em condições controladas, não sofreram alteração no período de avaliação. Entretanto, o mesmo pode não ocorrer no montante de resíduo acumulado no pátio da Cia. Ingá, tendo em vista que, nesse caso, deve ser considerada a ocorrência de chuvas, que poderiam causar variações nos valores de $\mathrm{pH}$;

- Quanto aos produtos industriais utilizados, os maiores valores de $\mathrm{pH}$ foram encontrados nas maiores doses dos Inertizantes 2 e 3 .

- Todos os inertizantes utilizados foram eficientes na redução da biodisponibilidade do Zn, sendo capazes de fazer o Zn passar de uma fração química menos estável para outra mais estável.

\section{REFERÊNCIAS}

ABNT (ASSOCIAÇÃO BRASILEIRA DE NORMAS TÉCNICAS). NBR-10007: amostragem de resíduos sólidos. Rio de Janeiro, 2004. 21p.

ANDRADE, J. C. M.; TAVARES, S. R. L.; MAHLER, C. F. Fitorremediação: o uso de plantas na melhoria da qualidade ambiental. 1.ed. São Paulo: Oficina de Textos, 2007. 176 p.

ARAÚJO, J. C. T. de; NASCIMENTO, C. W. A. do; FILHO, F. F. C. Disponibilidade de silício e biomassa de milho em solo contaminado por chumbo tratado com silicato, Ciência e Agrotecnologia, Lavras: Editora UFLA, v. 35, n. 5, p. 878-883, set-out./2011.

AUGUSTO, A.; YAMAMOTO, F. S.; PITTA, N. S. Análise da Área de Bota-Fora de Resíduos Industriais Depositados na Cidade de Volta Redonda - Estudo de Caso Rodovia do Contorno. 2012. 70 f. Monografia (Especialização) - Curso de Engenharia Ambiental. Centro Universitário de Volta Redonda UniFOA. 2012.

BORGES, M. R.; COUTINHO, E. L. M. Metais pesados do solo após aplicação de biossólidos. I Fracionamento. Seção IX - Poluição do solo e qualidade ambiental. Revista Brasileira de Ciência do Solo, v.28, p.543-555, 2004.

BORGES JUNIOR, M.; MELLO, J. W. V.; SCHAEFER, C. E. G. R.; DUSSIN, T. M.; JUNIOR, J. A. Distribuição e formas de ocorrência de zinco em solos no município de Vazante - MG. Revista Brasileira de Ciência do Solo, v.32, p.2183-2194, 2008.

DE OLIVEIRA, R. C. B.; MARINS, R. V. Dinâmica de Metais-Traço em Solo e Ambiente Sedimentar Estuarino como um Fator Determinante no Aporte desses Contaminantes para o Ambiente Aquático: Revisão. Revista Virtual de Química, v.3, n.2, p.88-102, abr-jun./2011.

DO NASCIMENTO, Robervone S. de M. P.; SKORUPA, Alba L. A.; PASSOS, Leônidas P.; MARQUES, João J. Extração e fitodisponiblidade de zinco e chumbo em latossolo tratado com resíduos de siderurgia, Revista Brasileira de Ciências Agrárias, Recife: UFRPE, v.9, p.322-329, 2014.

KELLER, C.; VÉDY, J. C. Distribution of cadmium fractions in two forest soils. Journal Environmental Quality, v.23, p.987-999, 1994. 
KWON, M.J.; BOYANOV, M.I.; YANG, J.; LEE, S.; HWANG, Y.H.; LEE, J.Y.; MISHRA, B.; KEMMER, K.M. Transformation of zinc-concentrate in surface and subsurface environments: Implications for assessing zinc mobility/toxicity and choosing an optimal remediation strategy. Environmental Pollution, v.226, p.346-355, 2017.

LEE, A. Y. W.; LIM, S. F.; CHUA, S. N. D.; SANAULLAH, K.; BAINI, R.; ABDULLAH, M. O. Adsorption Equilibrium for Heavy Metal Divalent lons $\left(\mathrm{Cu}^{2+}, \mathrm{Zn}^{2+}\right.$, and $\left.\mathrm{Cd}^{2+}\right)$ into Zirconium-Based Ferromagnetic Sorbent. Advances in Materials Science and Engineering, vol. 2017, Article ID 1210673, 13 pages, 2017. doi:10.1155/2017/1210673

LEPSCH, Igo F. Formação e conservação dos solos. 2.ed. São Paulo: Oficina de Textos, 2010. 216p.

LINDSAY, W.L., NORWELL, W. A. Development of a DTPA test zinc, iron, manganese and copper, Soil Science Society of America, v.42, p.421-428, 1978.

MARQUES, R. F. P. V. Impactos ambientais da disposição de resíduos sólidos urbanos no solo e na água superficial em três municípios de Minas Gerais. 2011. 95 f. Dissertação (Mestrado) - Universidade Federal de Lavras, 2011.

MELO, É. E. C.; NASCIMENTO, C. W. A. do; SANTOS, A. C. Q. Solubilidade, fracionamento e fitoextração de metais pesados após aplicação de agentes quelantes. Revista Brasileira de Ciência do Solo, Recife, v.30, 1051-1060 p., 2006.

MILLER, W. P.; MARTENS, D. C., ZELAZNY, L. W.; KORNEGAY, E. T. Forms of solid phase copper enriched swine manure, Journal Environmental Quality, v.15, p.69-72, jan/1986.

NASCIMENTO, C. W. A.; FONTES, R. L. F.; NEVES, J. C. L.; MELÍCIO, A. C. F. D. Fracionamento, dessorção e extração química de zinco em latossolos. Revista Brasileira de Ciência do Solo, v.26, p.599-606, 2002.

RODRIGUES, A. C. D. Utilização de Brachiaria decumbens na Fitoestabilização de Solos Contaminados por Metais Pesados Provenientes da Destruição de Munição. 2010. 67 f. Dissertação (mestrado) Instituto de Agronomia. Universidade Federal Rural do Rio de Janeiro, 2010.

SANTOS, G. C. G. Comportamento de B, Zn, Cu, Mn e Pb em solo contaminado sob cultivo de plantas e adição de fontes de matéria orgânica como amenizante do efeito tóxico. Piracicaba, Universidade de São Paulo, Escola Superior de Agricultura, 2005. 153p. (Tese de Doutorado)

SIMÃO, J.B.P.; SIQUEIRA, J. O. Solos contaminados por metais pesados: Características, implicações e remediação, Informe agropecuário, v.22, n.210, p.18-26, 2001.

SPOSITO, G. The chemistry of soils, New York: Oxford University Press, 2008. 330p.

TELES, A. G. N.; FERREIRA, R. M. Sustentabilidade e uso da água nas atividades agrárias e cultura desenvolvimentista. Revista da Faculdade de Direito do Sul de Minas, v.32, n.2, p.299-317, 2017.

UDDIN, M. K. A review on the adsorption of heavy metals by clay minerals, with special focus on the past decade. Chemical Engineering Journal, v.308, p.438-462, 2017.

URE, A.; QUEVAUVILLER, P. H.; MUNTAU, H. \& GRIEPINK, B. Speciation of heavy metals in soils and sediments, an account of the improvement and harmonization of extraction techniques undertaken 
under the auspices of the BCR of the Commission of the European Communities. International Journal of Environmental Analytical Chemistry, v.51, p.135-151, 1993.

XU, L.; CUI, H.; ZHENG, X.; ZHOU, J.; ZHANG, W. LIANG, J.; ZHOU, J. Changes in the heavy metal distributions in whole soil and aggregates affected by the application of alkaline materials and phytoremediation. RSC Advances, v.7, p.41033-41042, 2017.

ZHOU, T.; WU, L.; LUO, Y.; CHRISTIE, P. Effects of organic matter fraction and compositional changes on distribution of cadmium and zinc in long-term polluted paddy soils. Environmental Pollution, v.232, p.514-522, 2018.

ZHU, Q. H.; HUANG, D. Y.; LIU, S. L.; LUO, Z. C.; ZHU, H. H.; ZHOU, B.; LEI, M.; RAO, Z. X., CAO, X. L.. Assessment of single extraction methods for evaluating the immobilization effect of amendments on cadmium in contaminated acidic paddy soil, Plant Soil Environmental, v.58, p.98-103, 2012. 